\title{
CANONICAL TRANSFORMATIONS FOR SPACE TRAJECTORY OPTIMIZATION
}

\author{
Christine M. Haissig* and Kenneth D. Mease ${ }^{* *}$ \\ Department of Mechanical and Aerospace Engineering \\ Princeton University \\ Princeton, New Jersey 08544 \\ and \\ Nguyen X. Vinh ${ }^{\dagger}$ \\ Department of Aerospace Engineering \\ University of Michigan \\ Ann Arbor, Michigan 48109
}

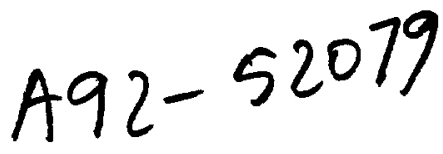

\begin{abstract}
Optimal space trajectory problems and the necessary and sufficient conditions that define their solutions are stated most compactly in terms of position and velocity vectors. To obtain analytical or numerical solutions, however, the problems are expressed using a particular set of coordinates. Each set of coordinates has advantages and disadvantages depending on the application. Thus, it may be useful to be able to transform from one set of coordinates to another during the course of solving an optimization problem. If the problem has been formulated using adjoint coordinates, the transformation requires not only transformation of the state coordinates, which are well-known, but also transformation of the adjoint coordinates. This combined transformation of the state and adjoint must be a canonical transformation for the extremal trajectory generated with the new coordinates to be the same as the extremal trajectory generated with the old coordinates.

In this paper, the canonical transformations between four common sets of coordinates used in space trajectory optimization-trajectory variables, orbit elements, equinoctial elements, Cartesian coordinates-are developed for the coplanar case. Applications of the canonical transformations in numerical optimization and the development of the necessary conditions for optimality are discussed.
\end{abstract}

\section{Introduction}

Optimal space trajectory problems, such as the minimumfuel transfer problem for a spacecraft in a central inverse square gravity field, and the necessary and sufficient conditions that define their solutions are stated most compactly in terms of position and velocity vectors [Mar79]. To obtain analytical or numerical solutions, the problems are expressed using a particular set of coordinates. The Cartesian coordinates, trajectory variables, orbital elements, and equinoctial elements are four common sets of coordinates for space trajectory problems. Cartesian coordinates have heen used for the analytical solution of optimal high thrust orbit transfer problems [Law63, Haz70] and the numerical solution of optimal low thrust transfer problems [Bar88, Enr90, Red84]. Orbital elements have been used to develop approximate solutions for optimal low thrust problems, using averaging [Ede65, Mar77,

\footnotetext{
* Graduate Student, Member

** Assistant Professor, Associate Fellow

† Professor, Member

Copyright 1992 by the Americal Institute of Aeronautics and Astronautics, Inc. All rights reserved.
}

Mar81] and linearization [Ede64, Ede66]. To avoid the singularities of the classic orbital elements, the equinoctial elements [Bro72, Bat87] have been used to develop a solution to the linearized rendezvous problem [Gob65]. Trajectory variables have been used for the approximate and numerical solution of optimal low thrust transfer problems [Ros61, Bro91, Mea90].

Each of these sets of coordinates has both attractive and unattractive features for analysis and computation. The coordinates that are best for analytical solutions such as averaging may not be best for numerical optimization. Therefore, it is advantageous to convert between sets of coordinates when solving optimal space trajectory problems. This conversion requires not only transformations of the state variables, which are well-known, but also transformations of the adjoint or costate variables. The combined transformation of the state and adjoint must be a canonical transformation for the extremal trajectory generated in the new coordinates to be the same as the extremal trajectory generated in the old coordinates.

One common purpose for using a canonical transformation is to increase the number of ignorable coordinates in a Hamiltonian system, which inakes the differential equations easier to solve. Fraejis de Veubeke ${ }^{1}$ used a canonical transformation in the minimum-fuel transfer problem for a thrust-limited rocket to transform the optimal steering control to a state variable when investigating the case of intermediate-thrust extremals [Fra65]. Marec and Vinh used a canonical transfornation to change the independent variable from time to characteristic velocity when solving the minimum-fuel, impulsive thrust problem [Vin70]. Marec developed the general minimum-fuel transfer problem using orbit elements with a three-dimensional canonical transformation from Cartesian coordinates to orbit elements [Mar79].

In this paper, canonical transformations between the four sets of coordinates commonly used in coplanar trajectory optimization-Cartesian coordinates, trajectory variables, orbital elements, and equinoctial elements-are developed. These canonical transformations are composed of state and adjoint coordinate transformations. The state transformations, which are independent of the adjoint transformations, are well-known, so the focus is on determining the corresponding adjoint transformations such that the combined state and adjoint transformation is canonical. The canonical transformations

\footnotetext{
${ }^{1}$ Marec credits Fraejis de Veuheke as the first person to apply canonical transformation systematically to the problem of optimal transfers in [Mar79]. pp. 46
} 
between the orbit elements and trajectory variables [Hai91] and between the Cartesian coordinates and orbital elements [Mar79] have been developed previously but are considered here for completeness. Following the derivation of the canonical transformations, specific applications of these canonical transformations for space trajectory optimization problems are presented.

\section{Hamiltonian System for an Optimal Trajectory}

The Mayer form of the general optimal control problem is to determine the $m$-dimensional piecewise-continuous control function $u$ on the time interval $\left[t_{0}, t_{f}\right]$ that drives the $n$ dimensional state $\mathbf{x}$ from an initial value $\mathbf{x}_{0}$ to a final value $\mathbf{x}_{\mathrm{f}}$, or more generally to a final manifold defined by the k-dimensional $(k \leq n)$ constraint vector $\psi\left(x\left(t_{f}\right), t_{f}\right)=0$, and minimizes the performance index

$$
\mathrm{J}=\Phi\left(\mathbf{x}\left(\mathrm{t}_{\mathbf{f}}\right), \mathrm{t}_{\mathbf{f}}\right)
$$

subject to the state equations (differential constraints)

$$
\dot{x}(t)=f(t, x(t), u(t))
$$

and the control constraint

$$
\mathbf{u}(t) \in U(t)
$$

for $t \in\left[t_{0}, t_{f}\right]$ and $U(t)$ a closed subset of $\mathscr{R}^{\mathrm{m}}$.

In space trajectory optimization [Mar79], the control is the thrust or thrust acceleration vector, and the state is comprised of the position vector $\mathbf{r}$, velocity vector $\mathbf{v}$, and the mass $m$. For the minimum-fuel problem, the performance index to be minimized is $J=-m\left(t_{f}\right)$. The state equations are

$$
\dot{r}(t)=v(t) ; \quad \dot{v}(t)=g(r, t)+\frac{T(t)}{m(t)} ; \dot{m}(t)=-\frac{T(t)}{g_{s} I_{s p}}
$$

where $\mathrm{g}$ is the gravitational acceleration and $\mathrm{g}_{\mathrm{s}}$ is its magnitude at sea level, $T$ is the thrust vector and $T$ is its magnitude, and $I_{s p}$ is the specific impulse. In special cases, it is advantagous 10 replace the mass with another coordinate. If the propulsion system is assumed to have constant ejection velocity, the characteristic velocity, or the time integral of the thrust acceleration magnitude, is used in place of mass; if the propulsion system is assumed to have limited power, the time integral of the thrust acceleration magnitude squared is used in place of mass. For the planar problem, the state $x=(r, v, m)^{T}$ is five-dimensional, since $\mathbf{r}$ and $\mathbf{v}$ each have dimension 2 .

If $\mathbf{u}^{*}$ is the optimal control and $\mathbf{x}^{*}$ the corresponding optimal trajectory and assuming the problem is normal, it is necessary [Fle75] that a nonzero $k$-dimensional constant vector $\mu$ and a nonzero $n$-dimensional adjoint vector function $\mathbf{p}(t)$ exist such that

$$
\begin{aligned}
& \dot{\mathbf{p}}(\mathrm{t})=\left(\frac{\partial}{\partial \mathbf{x}} \mathbf{f}\left(\mathrm{t}, \mathbf{x}^{*}(\mathrm{t}), \mathbf{u}^{*}(\mathrm{t})\right)\right)^{\mathrm{T}} \mathbf{p}(\mathrm{t}) \\
& \mathbf{u}^{*}(\mathrm{t})=\arg \max \left(\mathbf{p}^{\mathrm{T}}(\mathrm{t}) \mathbf{f}\left(\mathrm{t}, \mathbf{x}^{*}(\mathrm{t}), \mathbf{u}(\mathrm{t})\right)\right) \\
& \mathbf{p}^{\mathrm{T}}\left(\mathrm{t}_{\mathrm{f}}\right)=\frac{\partial \Phi}{\partial \mathbf{x}}\left(\mathbf{x}^{*}\left(\mathrm{t}_{\mathrm{f}}\right), \mathrm{t}_{\mathrm{f}}\right)+\mu^{\mathrm{T}} \frac{\partial \psi}{\partial \mathbf{x}}\left(\mathbf{x}^{*}\left(\mathrm{t}_{\mathrm{f}}\right), \mathrm{t}_{\mathrm{f}}\right)
\end{aligned}
$$

and

$$
p^{T}\left(t_{f}\right) f\left(t_{f}, x^{*}\left(t_{f}\right), u^{*}\left(t_{f}\right)\right)=\frac{\partial \Phi}{\partial t}\left(x^{*}\left(t_{f}\right), t_{f}\right)+\mu^{T} \frac{\partial \psi}{\partial t}\left(x^{*}\left(t_{f}\right), t_{f}\right)
$$

These conditions along with the state equations (2) and the boundary conditions

$$
\begin{aligned}
& x^{*}\left(t_{0}\right)=x\left(t_{0}\right) \\
& \psi\left(x^{*}\left(t_{f}\right)\right)=0
\end{aligned}
$$

constitute a two-point boundary value problem, the solutions of which are candidates for locally minimizing the performance index. The state trajectory corresponding to a solution of the two-point boundary-value problem is called an extremal trajectory. Equations (7) and (8) are known as the transversality conditions.

Assuming an explicit solution of eq. (6) of the form

$$
\mathbf{u}^{*}=\mathrm{g}\left(\mathrm{t}, \mathrm{x}^{*}, \mathbf{p}\right)
$$

the optimal Hamiltonian $\mathrm{H}^{*}$ is defined by

$$
H^{*}=p^{T}(t) f\left(t, x^{*}(t), g\left(t, x^{*}, p\right)\right) .
$$

The state and adjoint differential equations can be expressed as a canonical Hamiltonian system,

$$
\begin{aligned}
& \dot{x}^{*}=\left(\frac{\partial \mathrm{H}^{*}}{\partial \mathbf{p}}\right)^{\mathrm{T}} \\
& \dot{\mathbf{p}}=-\left(\frac{\partial \mathrm{H}^{*}}{\partial \mathbf{x}^{*}}\right)^{\mathrm{T}} .
\end{aligned}
$$

In the case of space trajectory design, $p=\left(p_{r}{ }^{T}, p_{v}{ }^{T}, p_{m 1}\right)^{T}$. The qualifier "* " is suppressed from here on.

\section{Canonical Transformation Theory}

Because the state and adjoint equations of the optimal control problem comprise a Hamiltonian system, the powerful canonical transformation theory of mechanics can be applied. A canonical transformation introduces no new physics to a problem, but it may facilitate analysis or physical interpretation of the motion, whose underlying properties are the same in the old and new coordinates [Lic83]. One use of canonical transformations is to increase the number of ignorable coordinates, thus simplifying the integration of the Hamiltonian system. In the optimal control context, canonical transformations allow one to translate the necessary conditions, integrals of the motion, approximate analytical solutions, etc., derived in terms of one set of coordinates into the equivalent results in terms of another set of coordinates, avoiding the need for re-derivation.

Let $\mathbf{x}^{A}$ and $\mathbf{p}^{A}$ denote the state and adjoint coordinate vectors for coordinate set $A$, and $x^{B}$ and $p^{B}$ denote those for coordinate set $B$. In the transformations of interest here, the transformation between the state coordinate vectors are timeindependent and do not involve the adjoint coordinate vectors; thus they are of the form 


$$
\mathbf{x}^{\mathrm{B}}=\phi_{A}^{\mathrm{B}}\left(\mathbf{x}^{\mathrm{A}}\right)
$$

and correspond to time-independent Lagrangian point transformations of mechanics [Lan49]. In the case of a Lagrangian point transformation, the Hamiltonian is an invariant of the associated canonical transformation from $A$ coordinate vectors $\left(\mathbf{x}^{\mathrm{A}} ; \mathbf{p}^{\mathrm{A}}\right)$ to $\mathrm{B}$ coordinate vectors $\left(\mathbf{x}^{\mathrm{B}} ; \mathbf{p}^{\mathrm{B}}\right)$ [Lan 49$]$,

$$
\mathbf{p}^{\mathrm{A}} \cdot \mathrm{d} \mathbf{x}^{\mathrm{A}}=\mathbf{p}^{\mathrm{B}} \cdot \mathbf{d} \mathbf{x}^{\mathrm{B}} .
$$

It follows that the adjoint transformation

$$
\mathbf{p}^{B}=\left[\left[\frac{\partial \phi_{A}^{B}}{\partial \mathbf{x}^{A}}\right]^{T}\right]^{-1} \mathbf{p}^{A}=T_{A}^{B} \mathbf{p}^{A}
$$

and the transformation of eq. (15) form a canonical transformation $C_{A}^{B}=\left[\phi_{A}^{B}, T_{A}^{B}\right]:\left(x^{A} ; p^{A}\right) \rightarrow\left(x^{B} ; \mathbf{p}^{B}\right)$.

A set of transformations together with the composition operation constitute a group if the following four properties are satisfied [Gol59]:

(i) The set contains the identity transformation.

(ii) The inverse of each element of the set exists and is a member of the set.

(iii) The set is closed under composition.

(iv) The composition operation is associative.

The canonical transformations associated with point transformations form a group on regions of the abstract state space where the point transformations are one-to-one and onto (injective). Since our interest is transformations between any two of the four sets of state coordinates, there are twelve canonical transfornations to be detennined. Given the group structure it is sufficient to develop the three canonical transformations from the Cartesian coordinates to the trajectory variables, the orbital elements to the trajectory variables, and the equinoctial elements to the orbital elements; the others can be obtained from composition and inversion of these. Since the state coordinate transformations are known, it is the adjoint coordinate transformations that are of interest here. Explicitly, if

$$
C_{A}^{B}=\left[\phi_{A}^{B}, T_{A}^{B}\right]
$$

and

$$
C_{B}^{D}=\left[\phi_{B}^{D}, T_{B}^{D}\right]
$$

then

$$
C_{A}^{D}=\left[\phi_{A}^{D}, T_{A}^{D}\right]=\left[\phi_{B}^{D} \phi_{A}^{B}, T_{B}^{D} T_{A}^{B}\right]
$$

and

$$
C_{B}^{A}=\left[\phi_{B}^{A}, T_{B}^{A}\right]=\left[\left(\phi_{A}^{B}\right)^{-1},\left(T_{A}^{B}\right)^{-1}\right]
$$

Some of the state coordinate sets considered here are not well-defined for certain points in the abstract state space; for example, one of the orbital elements is not well-defined for a circular orbit. The related singularities in the transformations at such points destroy the group structure; in particular, certain inverse transformations may not exist. Therefore, care must be taken in applying the canonical transformations developed in this paper.

\section{Definition of the State Coordinates}

The Cartesian coordinates, trajectory variables, orbital elements, and equinoctial elements must be defined before the canonical transformations for the coplanar transfer problem can be developed. In Cartesian coordinates, $\mathbf{r}$ is expressed using $\mathbf{x}$ and $y$, where $x$ is oriented along the line of nodes (or another reference line in the case of an equatorial orbit), and $\mathbf{v}$ is expressed using $v_{x}$ and $v_{y}$, where $v_{x}$ is also along the line of nodes (fig. 1). The corresponding adjoint coordinates are $\mathrm{p}_{\mathrm{x}}$, $\mathrm{p}_{\mathbf{y}}, \mathrm{P}_{\mathrm{x}}$, and $\mathrm{p}_{\mathrm{v}_{\mathrm{y}}}$. In trajectory variables, $\mathbf{r}$ is expressed using the radial position $r$ and the polar angle $\theta$, where $\theta$ is measured from the line of nodes (fig. 1). $v$ is expressed using the speed $v$ and the flight path angle $\gamma$, which measures the angle between the velocity vector and local horizontal. The corresponding adjoint coordinates are $\mathrm{p}_{\mathrm{r}}, \mathrm{p}_{\theta}, \mathrm{p}_{\mathrm{v}}$, and $\mathrm{p}_{\gamma}$.

Two sets of orbital elements are used (fig. 2). The first set consists of the semimajor axis a, eccentricity e, argument of periapse $\omega$, and mean anomaly $M$, with the corresponding adjoint coordinates $\mathrm{Pa}, \mathrm{Pe}_{\mathrm{e}}, \mathrm{p}_{(1)}$, and $\mathrm{pM}_{\mathrm{M}}$. In the second set, the true anomaly $\mathrm{f}$ replaces $\mathrm{M}$; the corresponding adjoint coordinates are $p_{a}^{\prime}, p_{e}^{\prime}, p_{b}^{\prime}$, and $p_{f}^{\prime}$, where the primes have been added to avoid confusion with the adjoint coordinates $\mathrm{Pa}_{\mathrm{a}}, \mathrm{p}_{\mathrm{e}}, \mathrm{P}_{\mathrm{o}}$, and $\mathrm{p}_{\mathrm{M}}$. Two sets of equinoctial elements are also considered. The coordinate names follow the convention of Battin [Bat87]. The first set is a, $P_{1}$ and $P_{2}$, which are computed from $e$ and $\omega$, and the mean longitude 1 ; the corresponding adjoint coordinates are $\mathrm{p}_{\mathrm{a}}, \mathrm{pP}_{1}, \mathrm{pP}_{2}$, and $\mathrm{p}_{\mathrm{J}}$. In the second set, the true longitude $\mathrm{L}$ replaces 1 and $p_{L}$ replaces $p$. The explicit relations between the orbital elements and the equinoctial elements are given in the next section.

All of the state and adjoint coordinates are nondimensionalized using the reference position $r_{r e f}$ and the reference time $t_{\text {ref }}$, where

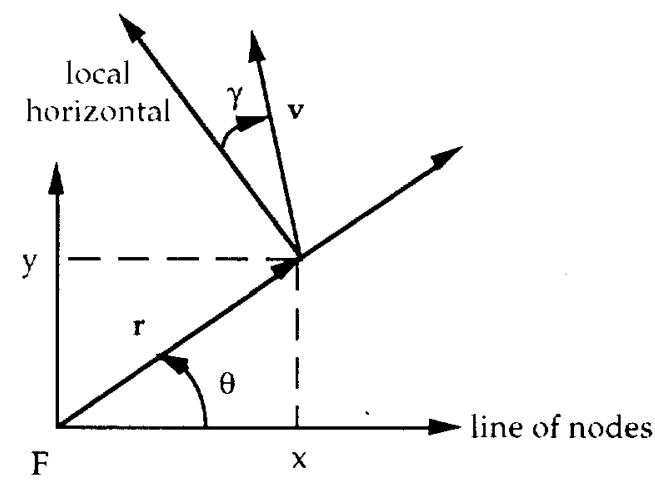

Fig. 1. Description of the Cartesian Coordinates and Trajectory Variables. $F$ is the focus of the orbit and the center of the central body 


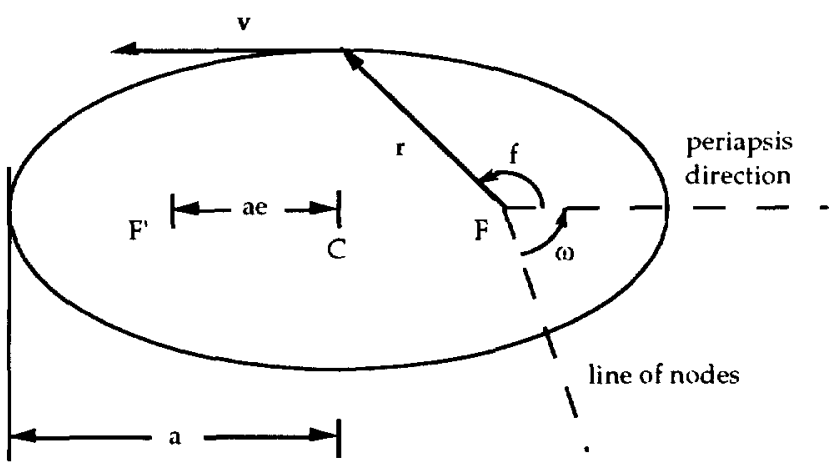

Fig. 2. Description of the Orbital Elements. $F$ is the focus of the orbit and the center of the central body. $F^{\prime}$ is the vacant focus.

$$
\mathrm{t}_{\mathrm{ref}} \equiv \sqrt{\mu / \mathrm{r}_{\mathrm{ref}}^{3}}
$$

and $\mu$ is the gravitational constant of the central body. The nondimensionality is only apparent in the transformation between the orbital elements and trajectory variables, where $\mu$ does not appear in the state and adjoint transformations. Converting the canonical transformations that are presented in this paper to dimensional form is straightforward. For example, if $R$ is the dimensional radial position and $p_{R}$ the corresponding adjoint coordinate, then

$$
\mathrm{R}=\mathrm{r}_{\mathrm{ref}} \mathrm{r}
$$

and, using eqs. (15) and (17),

$$
\mathrm{pR}_{\mathrm{R}}=\mathrm{pr} / \mathrm{r}_{\mathrm{ref}} \text {. }
$$

\section{State Transformations}

The transformations between the state coordinates are well-known. To transform from the trajectory variables to the Cartesian coordinates (see fig. 1),

$$
\begin{aligned}
& x=r \cos \theta \\
& y=r \sin \theta \\
& v_{x}=v \sin (\gamma-\theta) \\
& v_{y}=v \cos (\gamma-\theta) .
\end{aligned}
$$

The transformation from classic orbital elements to equinoctial elements is [Bat87]

$$
\begin{aligned}
& P_{1}=e \sin \omega \\
& P_{2}=e \cos \omega \\
& 1=\omega+M
\end{aligned}
$$

$$
\mathrm{L}=\omega+\mathrm{f}
$$

or, conversely,

$$
\begin{aligned}
& \mathrm{e}^{2}=\mathrm{P}_{1}^{2}+\mathrm{P}_{2}^{2} \\
& \tan \omega=\frac{\mathrm{P}_{1}}{\mathrm{P}_{2}} \\
& \mathrm{M}=1-\omega \\
& \mathrm{f}=\mathrm{L}-\omega .
\end{aligned}
$$

The transformation from the trajectory variables to the orbital elements is more involved [Bat71]. Introducing the nondimensional energy $\xi$ and semi-latus rectum $p$,

$$
\begin{aligned}
& \xi=-\frac{1}{2 a}, \\
& p=r^{2} v^{2} \cos ^{2} \gamma
\end{aligned}
$$

one transformation from the trajectory variables to orbital elements is

$$
\begin{aligned}
& \xi=\frac{v^{2}}{2}-\frac{1}{r} \\
& e=\sqrt{1+2 \xi p} \\
& \omega=\theta-f . \\
& f=\arctan \left[\frac{p \tan \gamma}{p-r}\right],
\end{aligned}
$$

where $\mathrm{f}$ is related to $\mathrm{M}$ through Kepler's equation and the eccentric anomaly $\mathrm{E}$,

$$
\begin{aligned}
& M=E-e \sin E \\
& \tan \frac{f}{2}=\sqrt{\frac{1+e}{1-e}} \tan \frac{E}{2} .
\end{aligned}
$$

\section{Adjoint Transformations}

Three canonical transformations are presented in this section - a transformation between the Cartesian coordinates and the trajectory variables, a transformation from the orbital elements to the trajectory variables, and a transformation between the equinoctial elements and the trajectory variables. Because the state transformations are known, it is the adjoint trunsformations that are of interest. There are two equivalent approaches to developing these transformations. In the first approach, the explicit state coordinate transformations are used in eq. (17) to develop the canonical transformation. In the second approach, the state coordinate transformations are used in the invariance definition of eq. (16) and the adjoint coordinate transformations can be extracted by matching the coefficients of the differential terms. In the second approach, the old coordinates can be implicit functions of the new coordinates, 
rather than explicit functions. Because of this, it is sometimes simpler to take the second approach rather than the first. For instance, taking the partial derivative of a relationship such as that in eq. (42), which involves an inverse tangent function with the corresponding concerns about the quadrants, is more complicated than dealing with the implicit relationship

$$
\tan f=\left[\frac{p \tan \gamma}{p-r}\right]
$$

The second approach is the one used in the derivation of the following canonical transformations.

\section{Cartesian Coordinates and Trajectory Variables}

First the canonical transformation from the Cartesian coordinates $\left(x, y, v_{x}, v_{y}, m ; p_{x}, p_{y}, p_{v_{x}}, p_{v_{y}}, p_{m l}\right)$ to the trajectory variables $\left(r, \theta, v, \gamma, m ; p_{r}, p_{\theta}, p_{v}, p \gamma, p_{m}\right)$ is developed. From the invariance of the Hamiltonian, eq. (16),

$$
\begin{aligned}
p_{x} d x+p_{y} d y+p v_{x} d v_{x}+p v_{y} d v_{y}+p_{m} d m \\
=p_{I} d r+p_{\theta} d \theta+p_{v} d v+p_{\gamma} d \gamma+p_{m} d m
\end{aligned}
$$

Taking the differential of the state coordinate transformations in eqs. (25-28),

$$
\begin{aligned}
& d x=\cos \theta d r-\sin \theta d \theta \\
& d y=\sin \theta d r+r \cos \theta d \theta \\
& d v_{x}=\sin (\gamma-\theta) d v+v \cos (\gamma-\theta)(d \gamma-d \theta) \\
& d v_{y}=\cos (\gamma-\theta) d v-v \sin (\gamma-\theta)(d \gamma-d \theta)
\end{aligned}
$$

Substituting these relationships into eq. (46) and matching the coefficients of the differential terms yields the transformation

$$
\begin{aligned}
& p_{r}=p_{x} \cos \theta+p_{y} \sin \theta \\
& p_{\theta}=x p_{y}-y p_{x}+v_{x} p_{v_{y}}-v_{y} p_{v_{x}} \\
& v_{p v}=v_{x} p_{v_{x}}+v_{y} p_{v_{y}} \\
& p_{\gamma}=v_{y} p_{v_{x}}-v_{x} p_{v_{y}} \\
& p_{m}=p_{m} .
\end{aligned}
$$

Since the state transformations for the position and velocity coordinates are independent of $\mathrm{m}$, the corresponding adjoint coordinates in eqs. (51-54) are also independent of $\mathrm{m}$, and the identity of eq. (55) holds. This identity holds for all of the canonical transformations considered in this paper, because all of the position and velocity state transformations are independent of $\mathrm{m}$, and it will no longer be stated explicitly.

The inverse of this transformation, using eq. (2l), is

$$
\begin{aligned}
& p_{x}=p_{r} \cos \theta-p_{\theta} \frac{\sin \theta}{r}-p_{\gamma} \frac{\sin \theta}{r} \\
& p_{y}=p_{r} \sin \theta+p_{\theta} \frac{\cos \theta}{r}+p_{\gamma} \frac{\cos \theta}{r}
\end{aligned}
$$

$$
\begin{aligned}
& p_{v_{x}}=p_{v} \sin (\gamma-\theta)+p_{\gamma} \frac{\cos (\gamma-\theta)}{v} \\
& p_{v_{y}}=p_{v} \cos (\gamma-\theta)-p_{\gamma} \frac{\sin (\gamma-\theta)}{v}
\end{aligned}
$$

\section{Equinoctial Elements and Orbital Elements}

The next canonical transformation developed is a transformation between the orbit elements and equinoctial elements. Two different transformations are considered, using different coordinates for the position in the orbit. In the first case, a canonical transformation between the orbital elements ( $a$, $\left.\mathrm{e}, \omega, \mathrm{M}, \mathrm{m} ; \mathrm{p}_{\mathrm{a}}, \mathrm{Pe}_{\mathrm{e}} \mathrm{p}_{()}, \mathrm{PM}, \mathrm{P}_{\mathrm{m}}\right)$ and the equinoctial elements (a, $\left.\mathrm{P}_{1}, \mathrm{P}_{2}, \mathrm{l}, \mathrm{m} ; \mathrm{Pa}_{\mathrm{a}} \mathrm{PP}_{1}, \mathrm{PP}_{2}, \mathrm{P}_{1}, \mathrm{P}_{\mathrm{m}}\right)$ is considered. From the invariance of the Hamiltonian, eq. (16),

$$
\begin{aligned}
P_{a} d a & +p_{e} d e+p_{\omega} d \omega+p_{M} d M+p_{m} d m \\
& =p_{a} d a+P_{P_{1}} d P_{1}+p_{P_{2}} d P_{2}+p_{l} d l+p_{m} d m
\end{aligned}
$$

Taking the differential of eqs. (33-35),

$$
\begin{aligned}
& e d e=P_{1} d P_{1}+P_{2} d P_{2} \\
& \sec ^{2} \omega d \omega=\frac{d P_{1} P_{2}-P_{1} d P_{2}}{P_{2}^{2}} \\
& d M=d l-d \omega .
\end{aligned}
$$

Solving eqs. (61) and (62) for $\mathrm{dP}_{1}$ and $\mathrm{dP}_{2}$,

$$
\begin{aligned}
& d P_{1}=\frac{P_{1}}{e} d e+\frac{P_{2}{ }^{3} \sec ^{2} \omega}{e^{2}} d \omega=\sin \omega d e+P_{2} d \omega \\
& d P_{2}=\frac{P_{2}}{e} d e-\frac{P_{1} P_{2}^{2} \sec ^{2} \omega}{e^{2}} d \omega=\cos \omega d e-P_{1} d \omega
\end{aligned}
$$

Substituting egs. (63-65) into the right hand side of eq. (60).

$$
\begin{aligned}
P_{a} d a+ & P_{e} d e+P_{0} d \omega+P M d M+P m d m=P_{a} d a \\
& +P_{P_{1}} \sin \omega d e+P_{P_{1}} P_{2} d \omega+P_{P_{2}} \cos \omega d e \\
& -P_{P_{2}} P_{1} d \omega+P_{1} d M-P I d \omega+P_{m} d m
\end{aligned}
$$

Combining like ternns,

$$
\begin{aligned}
& P_{a}=P_{a} \\
& P_{e}=P_{P_{1}} \sin \omega+P_{P_{2}} \cos \omega \\
& P_{(1)}=P_{P_{1}} P_{2}-P_{P_{2}} P_{1}-P_{1} \\
& P_{M}=P_{1} .
\end{aligned}
$$

Inverting eqs. $(68-70)$,

$$
P_{P_{1}}=p_{e} \sin \omega+\left(p_{(1)}+p_{M}\right) \frac{\cos \omega}{e}
$$




$$
\begin{aligned}
& \mathrm{p}_{\mathrm{P}_{2}}=\mathrm{p}_{\mathrm{e}} \cos \omega-\left(\mathrm{p}_{\omega}+\mathrm{p}_{\mathrm{M}}\right) \frac{\sin \omega}{\mathrm{e}} \\
& \mathrm{pl}_{\mathrm{l}}=\mathrm{p}_{\mathrm{M}} .
\end{aligned}
$$

The next canonical transformation of interest is between the $\left(\mathrm{a}, \mathrm{e}, \omega, \mathrm{f}, \mathrm{m} ; \mathrm{Pa}_{\mathrm{a}} \mathrm{Pe}_{\mathrm{P}} \mathrm{P}_{\omega}, \mathrm{Pf}, \mathrm{P}_{\mathrm{m}}\right)$ coordinates and the $\left(\mathrm{a}, \mathrm{P}_{1}\right.$, $\left.\mathrm{P}_{2}, \mathrm{~L}, \mathrm{~m} ; \mathrm{p}_{\mathrm{a}}, \mathrm{pP}_{1}, \mathrm{pP}_{2}, \mathrm{pL}_{\mathrm{L}}, \mathrm{p}_{\mathrm{m}}\right)$ coordinates. The development is almost identical to the previous transformation. For the Hamiltonian to be invariant,

$$
\begin{aligned}
p_{a} d a & +p_{e} d e+p_{\omega} d \omega+p_{M} d M+p_{m} d m \\
& =p_{a} d a+p_{1} d P_{1}+p_{2} d P_{2}+p_{L} d L+p_{m} d m
\end{aligned}
$$

Computing the differential of eq. (19)

$$
\mathrm{dL}=\mathrm{df}+\mathrm{d} \omega
$$

Substituting this relationship, along with the differentials from eqs. (61) and (62) into eq. (74), the relationship between the adjoint coordinates is

$$
\begin{aligned}
p_{a} d a+ & p_{e} d e+p_{\omega} d \omega+p_{f} d f+p_{m} d m=p_{a} d a \\
& +p_{1} \sin \omega d e+p p_{1} p_{2} d \omega+p p_{2} \cos \omega d e \\
& -p_{2} P_{1} d \omega+p_{L} d f+p_{L} d \omega+p_{m} d m
\end{aligned}
$$

Combining like terms,

$$
\begin{aligned}
& \mathrm{p}_{\mathrm{e}}=\mathrm{p}_{1} \sin \omega+\mathrm{p}_{2} \cos \omega \\
& \mathrm{p}_{\mathrm{C}}=\mathrm{p}_{1} \mathrm{P}_{2}-\mathrm{p}_{2} \mathrm{P}_{1}+\mathrm{p}_{\mathrm{L}} \\
& \mathrm{p}_{\mathrm{f}}=\mathrm{p}_{\mathrm{L}} .
\end{aligned}
$$

with eq. (67). Inverting eqs. (77-79),

$$
\begin{aligned}
& \mathrm{p}_{1}=\mathrm{p}_{\mathrm{e}} \sin \omega+\left(\mathrm{p}_{\omega}-\mathrm{p}_{\mathrm{L}}\right) \frac{\cos \omega}{\mathrm{e}} \\
& \mathrm{p}_{2}=\mathrm{p}_{\mathrm{e}} \cos \omega-\left(\mathrm{p}_{0}-\mathrm{p}_{\mathrm{L}}\right) \frac{\sin \omega}{\mathrm{e}} \\
& \mathrm{p}_{\mathrm{L}}=\mathrm{p}_{\mathrm{f}} .
\end{aligned}
$$

\section{Trajectory Variables and Orbital Elements}

Finally, canonical transformations from two sets of orbit elements to the trajectory variables are developed. The authors derived these canonical transformations previously [Hai91], so only the transformations themselves are included here. The first canonical transformation is between the coordinates $(r, \theta, v, \gamma$, m; $\left.\mathrm{Pr}, \mathrm{P}_{\theta}, \mathrm{Pv}_{\mathrm{v}}, \mathrm{P}_{\gamma}, \mathrm{P}_{\mathrm{m}}\right)$ and the coordinates $\left(\mathrm{a}, \mathrm{e}, \omega, \mathrm{f}, \mathrm{m} ; \mathrm{P}^{\prime} \mathrm{a}\right.$. $\left.p^{\prime}, p^{\prime}{ }_{(1)}, p_{1}^{\prime}, p_{I n}\right)$ :

$$
\begin{aligned}
& p_{r}=\frac{2 a^{2}}{r^{2}} p_{a}^{\prime}+\left[p_{e}^{\prime}-\frac{\left(p_{f}^{\prime}-p_{(\omega)}^{\prime}\right) \sin f}{e(e+\cos f)}\right] \frac{(e+\cos f)}{r} \\
& p_{\theta}=p_{(i)}^{\prime}
\end{aligned}
$$

$$
\begin{aligned}
p_{v}=2 & a^{2} v p_{a}^{\prime}+\left[p_{e}^{\prime}-\frac{\left(p_{f}^{\prime}-p^{\prime}(\omega) \sin f\right.}{e(e+\cos f)}\right] \frac{2(e+\cos f)}{v} \\
p_{\gamma}= & \frac{p_{e}^{\prime}\left(1-e^{2}\right) \sin f}{(1+e \cos f)} \\
& +\frac{\left(p^{\prime} f-p^{\prime}(1)\right)}{e(1+e \cos f)}[e(1+e \cos f)+(e+\cos f)] .
\end{aligned}
$$

Equations (83-86) are cumbersome to invert symbolically but straightforward to invert numerically as long as the eccentricity is nonzero.

The adjoint transformation for the canonical transformation between the coordinates $\left(a, e, \omega, f, m ; p_{a}^{\prime}, p_{e}^{\prime}\right.$. $\left.\mathrm{p}_{(1)}^{\prime}, \mathrm{p}^{\prime} \mathrm{f}, \mathrm{p}_{\mathrm{m}}\right)$ and the coordinates $\left(\mathrm{a}, \mathrm{e}, \omega, \mathrm{M}, \mathrm{m} ; \mathrm{p}_{\mathrm{a}}, \mathrm{p}_{\mathrm{e}}, \mathrm{p}_{\omega}\right.$, $\left.\mathrm{pM}, \mathrm{p}_{\mathrm{m}}\right)$ is:

$$
\begin{aligned}
& p_{a}=p_{a}^{\prime} \\
& p_{e}=p_{e}^{\prime}+p_{f}^{\prime}\left(\frac{\operatorname{sinf}}{1-e \cos E}+\frac{\operatorname{sinf}}{1-e^{2}}\right) \\
& p_{(1)}=p_{(1)}^{\prime} \\
& p_{M}=p_{f}^{\prime} \frac{\operatorname{sinf}}{\sin E(1-e \cos E)},
\end{aligned}
$$

Equations (87-90) are easily invertible. Using the fact that

$$
\frac{1-\mathrm{e} \cos \mathrm{E}}{1-\mathrm{e}^{2}}=\frac{1}{1+e \cos \mathrm{f}}
$$

the inverse of eqs. $(87-90)$ is

$$
\begin{aligned}
& \mathrm{p}_{e}^{\prime}=p e-p_{M} \sin E\left(\frac{2+e \cos f}{1+e \cos f}\right) \\
& p_{0}^{\prime}=p_{0} \\
& p_{f}^{\prime}=p_{M} \frac{\sin E(1-e \cos E)}{\sin f} .
\end{aligned}
$$

Combining these intermediate canonical transformations, the canonical transformation between the coordinates $(r, \theta, v, \gamma, \mathrm{m}$; $\left.p_{r}, p_{H}, p_{V}, p_{\gamma}, p_{m}\right)$ and the coordinates $\left(a, e, \omega, M, m ; p_{a}, p_{e}\right.$, $\left.\mathrm{P}_{\mathrm{o}}, \mathrm{PM}, \mathrm{p}_{\mathrm{m}}\right)$ is:

$$
\begin{aligned}
& p_{r}=\frac{2 a^{2}}{r^{2}} p_{a}+\left[p_{e}^{\prime}-\frac{\left(p_{f}^{\prime}-p_{Q}\right) \sin f}{e(e+\cos f)}\right] \frac{(e+\cos f)}{r} \\
& p_{\theta}=p_{(1)} \\
& p_{v}=2 a^{2} v p_{a}+\left[p_{e}^{\prime}-\frac{\left(p_{f}^{\prime}-p_{0}\right) \sin f}{e(e+\cos f)}\right] \frac{2(e+\cos f)}{v} \\
& P_{\gamma}=\frac{P_{e}^{\prime}\left(1-e^{2}\right) \sin f}{(1+e \cos f)} \\
& +\frac{\left(p_{f}-P_{(1)}\right)}{e(1+e \cos f)}[e(1+e \cos f)+(e+\cos f)]
\end{aligned}
$$




$$
\begin{aligned}
& \mathrm{p}_{\mathrm{e}}^{\prime}=\mathrm{p}_{\mathrm{e}}-\mathrm{p}_{\mathrm{M}} \sin \mathrm{E}\left(\frac{2+e \cos f}{1+e \cos f}\right) \\
& \mathrm{p}_{\mathrm{f}}^{\prime}=\mathrm{p}_{\mathrm{M}} \frac{\sin \mathrm{E}(1-\mathrm{e} \cos \mathrm{E})}{\operatorname{sinf}}
\end{aligned}
$$

Equations (95-100) are also cumbersome to invert symbolically but straightforward to invert numerically as long as the eccentricity is nonzero.

\section{Applications}

\section{Numerical Optimization}

One of the most important applications for these canonical transformations is in the numerical solution of space trajectory optimization problems. Computing optimal trajectories and controls is a two step process when using an iterative numerical optimization algoithm. The first step is initializing the numerical optimization algorithm; the second step is running the algorithm. Numerical optimization algorithms at best converge to local minima. The starting solution with which the algorithm is initialized must be within the domain of convergence of the local minimum of interest. Developing such a starting solution is often the most challenging and least systematic step in solving an optimal control problem. For indirect methods such as the shooting method one must provide starting initial adjoint coordinates; for direct methods such as the gradient method one must provide a starting optimal control profile.

Throughout the years, researchers have developed many approximate analytical solutions to space trajectory optimization problems, particularly finite thrust problems, which are difficult to solve numerically. These approximate solutions can be used as starting solutions for numerical optimization. With canonical transformations such as those developed in the previous sections, these approximate solutions can be used in numerical optimization problems regardless of the coordinates used in the numerical algorithm (fig. 3). This is particularly useful for multiple-revolution low thrust transfers, where it is difficult to guess the thrust profile, let alone the initial adjoint coordinates. The authors have used the average solution [Ede65, Mar77. Mar81], developed in terms of the orbit elements, in computing the trajectory variable solution of the minimum-fuel, LP transfer problem [Hai91]. Other potentially useful approximate solutions for space trajectory optimization are linearized solutions for low thrust propulsion using the LP model for rendezvous and transfer [Ede64, Gob64, Gob65] developed in terms of orbit elements for transfer and Cartesian coordinates for rendezvous, and the constant thrust, constant ejection velocity (C.EV) solution using an asymptotic expansion about the impulsive solution developed in terms of Cartesian coordinates [Kor71].

The advantages of using canonical transformation in conjunction with analytical solutions to do numerical optimization are numerous. A trajectory analyst can pick the best coordinates for numerical optimization independent of the coordinates used to develop the approximate solution. There is no need to develop new approximate solutions using different coordinates. Developing the candidate solution is systematic, and if the approximate solution is valicl for the transfer geometries and transfer times of interest, the optimization algorithm has a good chance of converging, and converging quickly. Although these analytical approximations require some numerical computation, this computation is insignificant relative to that required by just one iteration of a numerical optimization algorithm.

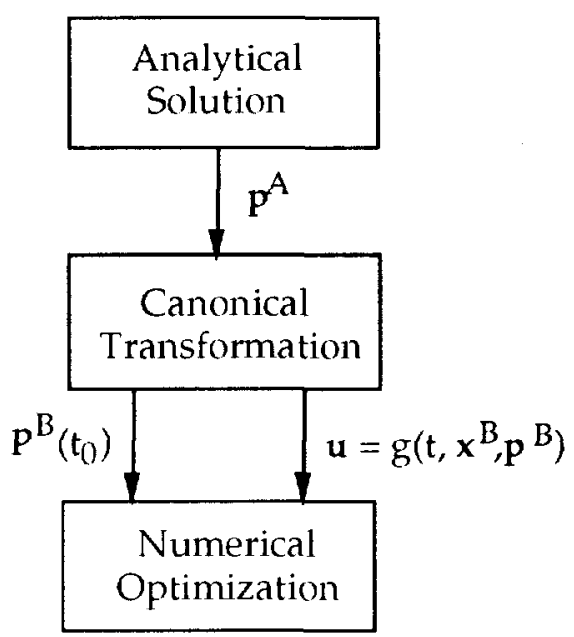

Fig. 3. Applying Approximate Solutions in Numerical Optimization Algorithms Using Canonical Transformation

\section{Transversality Conditions}

Another application of canonical transformation is in the analytical development of the optimal control problem. Once $\mathrm{H}^{*}$, the necessary and sufficient conditions, integrals of motion, and boundary conditions have been detemined in one set of coordinates, they can be expressed in another set using canonical transformation, obviating the need for re-derivation. This is particularly advantageous for the transversality conditions. If the final state constraints are complicated functions of the state coordinates, taking the partial derivatives with respect to the state coordinates and eliminating the Lagrange multipliers to form the transversality conditions can be algebraically cumbersome. However, these partial derivatives are an inherent part of the canonical transformations. For example, for a transfer between two orbits where the position in the final orbit is free

$$
\mathrm{M}=0
$$

or

$$
\mathrm{f}=0
$$

at the final time. The corresponding condition on the adjoint coordinates is

$$
\mathrm{PM}=0
$$

or

$$
p_{t}=0
$$

This condition can be written in terms of the equinoctial elements using the canonical transformations of egs. (73) and (78) as

$$
\text { PI. }=0
$$


or

$$
\mathrm{p}]=0 \text {. }
$$

In terms of trajectory variables, using eqs. (95-100), this condition is

$$
\operatorname{pr}_{\mathrm{r}} v \sin \gamma-\frac{\mathrm{p}_{\mathrm{v}} \sin \gamma}{\mathrm{r}^{2}}+\frac{\mathrm{p}_{\gamma} v \cos \gamma}{\mathrm{r}}\left(1-\frac{1}{\mathrm{r} \mathrm{v}^{2}}\right)+\frac{\mathrm{p}_{\theta} v \cos \gamma}{\mathrm{r}}=0
$$

In tenns of Cartesian coordinates, substituting eqs. (51-54) into eq. (107),

$$
p_{x} v_{x}+p_{y} v_{y}-p_{v_{x}} \frac{\cos \theta}{r^{2}}-p_{v_{y}} \frac{\sin \theta}{r^{2}}=0
$$

Similarly, when the orientation is free in the final orbit,

$$
p_{(1)}=0
$$

at the final time. Using at the canonical transfomation between the trajectory variables and the orbit elements, eq. (96), a free orientation implies that

$$
p_{\theta}=0
$$

In terms of equinoctial elements, from eq. (69) this transversality condition is

$$
\mathrm{p}_{\mathrm{P}_{1}} \mathrm{P}_{2}-\mathrm{p}_{\mathrm{P}_{2}} \mathrm{P}_{1}-\mathrm{p}_{1}=0
$$

or

$$
\mathrm{p}_{\mathrm{P}_{1}} \mathrm{P}_{2}-\mathrm{p}_{\mathrm{P}_{2}} \mathrm{P}_{1}+\mathrm{PL}=0
$$

Similarly, in terms of the Cartesian coordinates using eq. (53), the boundary condition is

$$
x p_{y}-y p_{x}+v_{x} p_{v_{y}}-v_{y} p_{v_{x}}=0
$$

All of these transversality conditions were derived without resorting to taking partial derivatives with respect to the state coordinates and eliminating the Lagrange multipliers.

\section{Conclusions}

Canonical transformations have been developed between the Cartesian coordinates, trajectory variables, equinoctial elements, and orbital elements for coplanar space trajectory optimization problems. The canonical transformations allow the state and adjoint equations or their solution, the optimal control, transversality conditions, and integrals of the motion, to be transformed between any of the common sets of coordinates for planar space trajectory optimization problems. Variations on the canonical transformations presented are straightforward to develop given the group properties of the canonical transformations.

These canonical transformations are useful in the numerical solution of finite thrust optimization problems, where the challenge is to determine an estimate of the initial adjoint or optimal control profile that is within the numerical algorithm's convergence domain for the global minimum. With the canonical transformations, approximate solutions to the finite thrust transfer problem can be used to initialize numerical optimization algorithms regardless of the coordinates chosen for the numerical algorithon.

\section{Acknowledgments}

This research is sponsored by the NASA Lewis Research Center under grant NAG3-915 and the Department of Defense through a National Defense Science and Engineering Graduate Fellowship.

\section{References}

[Bar88] Bartholomew-Biggs, M. C., L. C. W. Dixon, S. E. Hersom, and Z. A. Maany. 1988. The solution of some difficult problems in low-thrust interplanetary trajectory optimization. Optimal Control Applications and Methods 9: 229-51.

[Bat71] Bate, R. R., D. D. Mueller, and J. E. White. 1971. Fundamentals of astrodynamics. New York: Dover Publications.

[Bat87] Battin, R. H. 1987. An introduction to the mathematics and mathods of astrodynamics. New York: American Institute of Aeronautics and Astronautics.

[Bro72] Broucke, R. A., and P. J. Cefola. 1972. On the equinoctial orbit elements. Celestial Mechanics 5: 303-310.

[Bro91] Broucke, R. A. 1991. Low-thrust trajectory optimization in an inverse square force field. AAS 91159. AAS/AIAA Spaceflight Mechanics Meeting, Houston, Tex.

[Ede(A4] Edelbaum, T. N. 1964. Optimal low-thrust rendezvous and station keeping. AlAA Journal 2 (7): $1196-1201$.

[Ede65] Edelbaum, T. N. 1965. Optimum power-limited orbit transfer in strong gravity fields. AIAA Journal 3 (5): $921-25$.

[Ede66] Edelbaum, T. N. 1966. An asymptotic solution for optimum power limited orbit transfer. AIAA Journal $4(8): 1491-94$.

[Em90] Enright, P. J., and B. A. Conway. 1989. Discrete approximations to optimal trajectories using direct transcription and nonlinear programming. AIAA 902963. AIAA/AAS Astrodynamics Conference, Portland, Ore.

[Fle75] Fleming, W. H., and R. W. Rishel. 1975 Deferministic and stochastic optimal control. New York: Springer-Verlag.

[Fra65] Fraejis de Veubeke, B. 1965. Canonical transformations and the thrust-coast-thrust optimal transfer problem. Astronantica Acta 11 (4): 271-82.

[Gob64] Gobetz, F. W. 1964. Optimal variable-thrust transfer of a power-limited rocket between neighboring circular orbits. AIAA Journal 2 (2): 339-43.

[Gob65] Gobetz, F. W. 1965. A linear theory of optimum low-thrust rendezvous trajectories. Journal of the Astronatical Sciences 12 (3): 69-76. 
[Gol59] Goldstein, H. 1959. Classical Mechanics. Reading, Mass.: Addison-Wesley Publishing Co.

[Hai91] Haissig, C. M., K. D. Mease, and N. X. Vinh. 1991. Minimum-fuel, power-limited transfers between coplanar elliptical orbits. IAF-91-346. 42nd International Astronatical Federation Conference, Montreal, Canada.

[Haz70] Hazelrigg, G. A., and P. M. Lion. 1970. Analytical determination of the adjoint vector for optimum space trajectories. J. Spacecraft and Rockets 7 (10): 1200-7.

[Kor71] Kornhauser, A. L., P. M. Lion, and G. A. Hazelrigg. 1971. An analytic solution for constant-thrust, optimal-coast, minimum-propellant space trajectories. AIAA Journal 9 (7): 1234-39.

[Lan49] Lanczos, C. 1949. The variational principles of mechanics. Toronto: University of Toronto Press.

[Law63] Lawden, D.F. 1963. Optimal trajectories for space navigation. London: Butterworths.

[Lic83] Lichtenberg, A. J., and M. A. Lieberman. 1983. Regular and Stochastic Motion. New York: SpringerVerlag.

[Lue84] Luenberger, D. G. 1984. Linear and nonlinear programming. Reading, Mass.: Addison-Wesley Publishing Co.

[Mar77] Marec, J. P., and N. X. Vinh. 1977. Optimal lowthrust, limited power transfers between arbitrary elliptical orbits. Acta Astronautica 4: 511-40.

[Mar79] Marec, J. P. 1979. Optimal space trajectories. Amsterdam: Elsevier Scientific Publishing Co.

[Mar81] Marec, J. P., and N. X. Vinh. 1981. General study of optimal low thrust, limited power transfers between arbitrary elliptical orbits. European Space Agency Report ESA-TT-697.

[Mea90] Mease, K. D., C. M. Haissig, and N. X. Vinh. 1990. Comparison of solution approaches for minimum-fuel, low-thrust, power-limited orbit transfers. AIAA 90-2960. AIAA/AAS Astrodynamics Conference, Portland, Ore.

[Pow69] Powers, W. F., and B. D. Tapley. 1969. Canonical transformation: applications to optimal trajectory analysis. ALAA Joumal 7 (3): 394-99.

[Red84] Redding, D. C., and I. V. Breakwell. 1984. Optimal low-thrust transfers to synchronous orbit. J. Guidance $7(2): 148-55$.

[Ros61] Ross, S., and G. Leitmann. 1961. Low acceleration trajectory optimization in a strong central force field. IAS Symposium on Vehicle Systems optimization. Institute of Aerospace Sciences, New York, 127-37.

[Vin70] Vinh, N. X., and C. Marchal. 1970. Analytical solution of a class of optimum orbit modifications. Journal of Optimization Theory and Applications 5 (3): $178-96$ 